УДК 656.11:351.811.12

ОПРЕДЕЛЕНИЕ ХАРАКТЕРИСТИК ПОТОКА АВТОМОБИЛЕЙ ПРИ ОБЪЕЗДЕ МЕСТ ДОРОЖНО-ТРАНСПОРТНЫХ ПРОИСШЕСТВИЙ

\begin{abstract}
Наумова Н.А., Карачанская Т.А.
ФГБОУ ВО «Кубанский государственный технологический университет», Краснодар, e-mail: Nataly_Naumova@mail.ru

Актуальной задачей является моделирование транспортных потоков с целью выбора оптимальной схемы движения транспортных средств по улично-дорожной сети. Одной из проблем, влияющих на удобство передвижения по улично-дорожной сети, являются дорожно-транспортные происшествия. Задачей исследования является разработка математической модели и определение характеристик движения потока автомобилей при объезде мест дорожно-транспортных происшествий или дорожно-ремонтных работ. Разработана математическая модель движения автотранспортных средств в плотном транспортном потоке в случае, когда в результате дорожно-транспортного происшествия или ремонтных работ перекрывается движение по одной из полос. Интервалы по времени между автомобилями на каждой полосе движения приняты подчиненными закону Эрланга. Место инцидента представлено как система массового обслуживания. С помощью метода псевдосостояний и цепей Маркова рассчитаны характеристики движения автомобилей вблизи места дорожно-транспортного происшествия или дорожно-ремонтных работ. Приведен алгоритм корректировки данных о транспортных потоках в матрице корреспонденций сети. Моделирование движения транспортных потоков вблизи мест аварий позволяет прогнозировать вызванные этим задержки транспортных средств и корректировать оптимальные схемы организации движения. Результаты работы дополняют модель движения транспортных потоков, разработанную ранее авторами настоящей статьи.
\end{abstract}

Ключевые слова: математическая модель, организация движения, транспортный поток, система массового обслуживания, дорожно-транспортное происшествие

\title{
DETERMINATION OF VEHICLE FLOW CHARACTERISTICS WHEN BYPASSING ROAD ACCIDENT SITES
}

\section{Naumova N.A., Karachanskaya T.A.}

Kuban State Technological University, Krasnodar,e-mail: Nataly_Naumova@mail.ru

An urgent task is the modeling of traffic flows with the choice of the optimal scheme for the movement of vehicles along the road network. One of the problems affecting the convenience of movement on the road network is road accidents. The aim of the research is to develop a mathematical model and determine the characteristics of the traffic flow of cars when bypassing the places of road traffic accidents or road repair works. A mathematical model has been developed for the movement of vehicles in a dense traffic flow in the case when, as a result of a traffic accident or repair work, traffic on one of the lanes is blocked. The time intervals between cars on each lane are taken subordinate to Erlang's law. The scene of the incident is presented as a queuing system. Using the method of pseudo states and Markov chains, the characteristics of the movement of vehicles near the place of a traffic accident or road repair work are calculated. An algorithm for correcting data on traffic flows in the network correspondence matrix is presented. Modeling the traffic flow near the accident sites allows predicting the resulting vehicle delays and adjusting the optimal traffic management schemes. The results of the work complement the traffic flow model developed earlier by the authors of this article.

Keywords: mathematical model, traffic organization, traffic flow, queuing system, road traffic accident

Математическое моделирование движения автотраспортных средств в настоящее время имеет огромную область применения. Согласно требованиям Министерства транспорта РФ, математическое моделирование применяется при принятии управленческих решений на стратегическом, тактическом и опративном уровнях организации дорожного движения, так как это наиболее точный и удобный инструмент, позволяющий усовершенствовать существующую транспортную систему.

Сами математические модели транспортных потоков в настоящее время разделяют на макроскопические, мезоскопические и микроскопические. Макроскопическое моделирование устанавливает функциональные зависимости между отдельными показателями потока, например скоростью, плотностью и иинтенсивностью движения. При микроскопическом моделировании детально имитируется движение транспортных средств с целью установления показателей эффективности функционирования локального участка сети. Обычно имитационная модель базируется на статистическом распределении интервалов между транспортными средствами. Мезоскопическое моделирование служит «мостом» между микро- и макромоделями. Мезоскопические модели рассматривают единичные транспортные средства, но описывают их движение и взаимодействие на основании статистических зависимостей.

При решении отдельных задач может отдаваться предпочтение тому или иному методу моделирования, либо можно использовать комбинацию методов. 
Одной из проблем, влияющих на удобство передвижения по улично-дорожной сети, являются дорожно-транспортные происшествия (ДТП). Как правило, при ДТП перекрывается одна из полос для движения, образуется «узкое место», которое становится причиной заторов. Аналогичная ситуация может возникнуть при проведении дорожных работ.

Ввиду этого актуальной становится проблема моделирования движения автомобилей при объезде места дорожно-транспортного происшествия или дорожно-ремонтных работ.

Целью исследования является повышение качества организации движения транспортных потоков в случае дорожно-транспортного происшествия.

Задача исследования - разработка математической модели и определение характеристик движения потока автомобилей при объезде мест дорожно-транспортных происшествий или дорожно-ремонтных работ.

\section{Материалы и методы исследования}

При моделировании движения автомобилей в местах ДТП возможно применять различные типы моделирования.

Так, например, в работе [1] для моделирования ДТП использовались макроскопические переменные, такие как средняя плотность и средняя скорость. Данный подход достаточно упрощен, но позволяет прогнозировать влияние дорожно-транспортных происшествий на средние характеристики потоков. В работе [2] объединено стохастическое моделирование аварий с моделью Лайтхилла - Уизема - Ричардса (LWR). L. Jin и S. Amin в своей работе [3] представили модель динамики движения на автостраде при дорожно-транспортных происшествиях со стохастическим снижением пропускной способности. В их модели с помощью цепей Маркова моделируется сокращение пропускной способности дороги, определяются характеристики очереди, вызванной инцидентом на дороге. Использовать результаты работы [3] можно для анализа влияния стохастических колебаний пропускной способности на пропускную способность участка автострады.

Для описания модели движения автомобилей в местах ДТП можно также использовать подход авторов, описывающих транспортные потоки при пересечении блок-постов [4] или при движении через «бутылочное горло» [5].

В данной работе для моделирования движения автотранспортных средств вблизи места дорожно-транспортного происшествия будем использовать метод, относящийся к мезоскопическому моделированию. Базовые гипотезы и положения, характеризующие транспортные потоки, были описаны, например, в статье автора [6]. Транспортная сеть представляется в виде ориентированного графа. Необходимые сведения об организации движения, параметрах транспортных потоков содержатся в матрицах $A_{\text {STREETS }}$ и $B_{\text {INTERSECTION }}$ Информация о числе транспортных средств, проходящих от «источника» к «стоку» в течение времени $t$, содержится в OD-матрице (матрице корреспонденций) [7].

Распределение интервалов по времени в каждом из транспортных потоков по каждой из полос движения принято подчиненным распределению Эрланга. Данное распределение позволяет аппроксимировать с нужной степенью точности при правильном подборе параметров любое статистическое распределение [8]. Кроме того, такая гипотеза о распределении интервалов в случайном потоке позволяет с помощью метода псевдосостояний использовать цепи Маркова и определять характеристики функционирования системы массового обслуживания $[8,9]$.

\section{Результаты исследования и их обсуждение}

Рассмотрим движение автотранспортных средств в плотном транспортном потоке в случае, когда в результате дорожно-транспортного происшествия или ремонтных работ перекрывается движение по одной из полос.

1. Представление места ДТП как системы массового обслуживания

Будем рассматривать отрезок уличнодорожной сети между двумя соседними перекрестками, на котором произошло ДТП или ведутся ремонтные работы, как открытую систему массового обслуживания. Движение транспортных средств в плотном потоке, прибывающих по двум полосам к точке А (рис. 1), в данном случае замедляется. Согласно натурным наблюдениям, образуется общая очередь перед точкой A, несмотря на то, что автомобили располагаются на двух полосах. Дисциплина очереди - обслуживание в порядке прибытия транспортных средств (в порядке поступления заявок) FIFO. Количество мест в очереди ограничено количеством автомобилей, которые могут заполнить обе полосы от точки А до предшествующео узла сети (перекрестка) - точки С. Под временем обслуживания $T_{0}$ будем понимать среднее время (в секундах) объезда первым в очереди автомобилем препятствия $A B$. Интенсивность обслуживания $\mu=\frac{1}{T_{0}}$. 


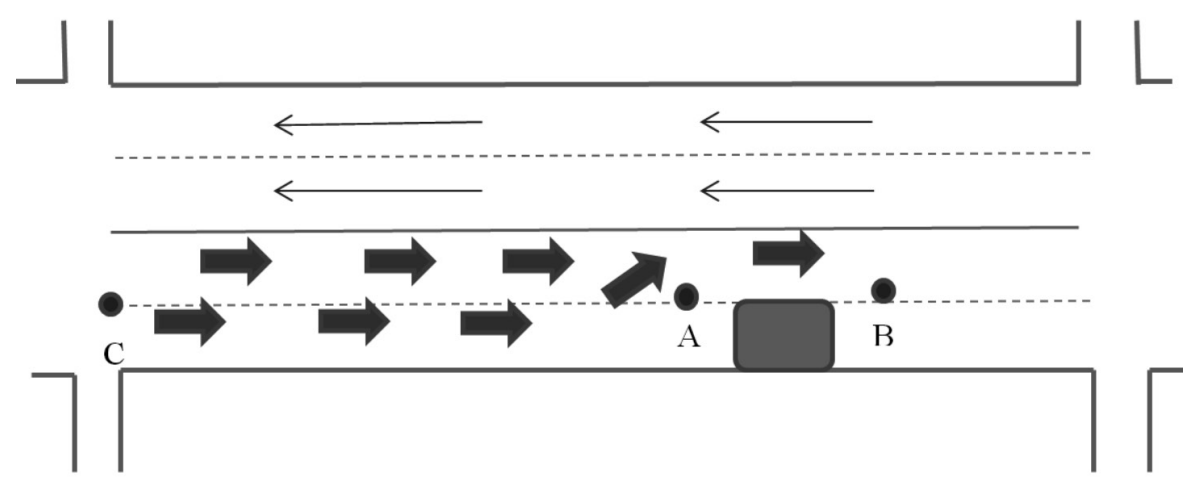

Рис. 1. Схема движения в месте ДТП

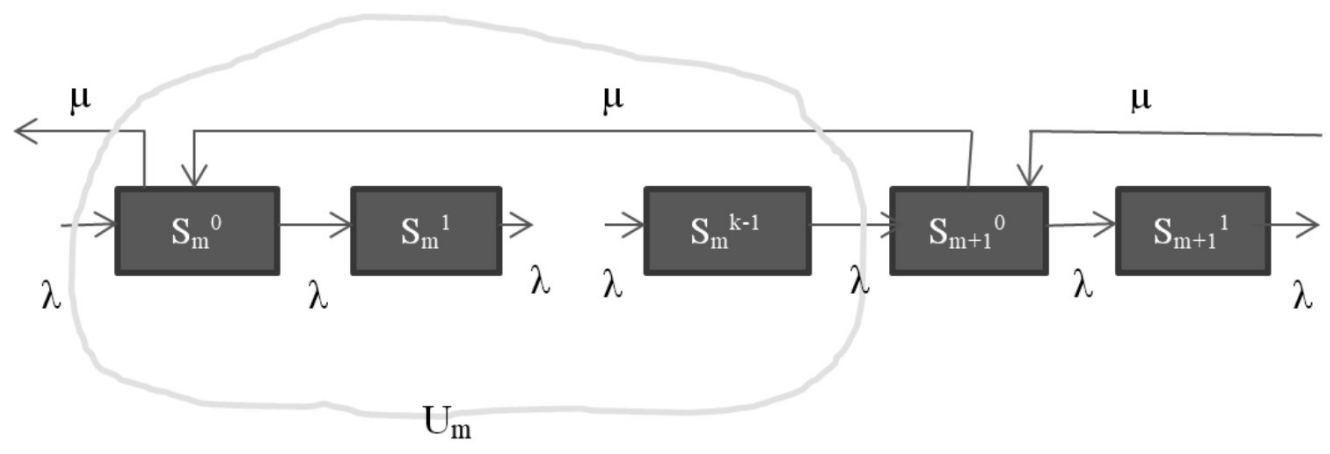

Рис. 2. Псевдосостояния модели системы массового обслуживания

2. Разработка математической модели движения автотранспортных средств в плотном потоке при ДТП

Согласно модели TIMeR_Mod интервалы по времени между транспортными средствами по каждой из полос распределены по закону Эрланга. Проведенные натурные обследования показали, что для плотного потока можно считать, что это специальный закон Эрланга порядка $k \geq 4$. Для расчетов параметров распределения Эрланга можно использовать результаты работы [10].

Пусть потоки П и $\Pi_{2}$ распределены по специальному закону Эрланга с параметрами $\lambda_{1}$ и $\lambda_{2}$ соответственно и с одинаковым значением параметра $k \geq 4$. Тогда «на обслуживание» прибывает поток $\Pi_{1+2}$ Пальма, распределение интервалов по времени между событиями в котором - это специальное распределение Эрланга такого же порядка $k$ с параметром $\lambda=\lambda_{1}+\lambda_{2}$. Время обслуживания будем считать распределенным по показательному закону с параметром $\mu=\frac{1}{T_{0}}$. В очереди могут находиться одновременно не более $N$ автомобилей, а в системе в целом - $(N+1)$ автомобиль: $N$ в очереди и один на обслуживании.

Для составления математической модели данной СМО воспользуемся методом псевдосостояний.

Опираясь на граф состояний (рис. 2), составим систему дифференциальных уравнений Колмогорова, описывающих пребывание СМО в псевдосостояниях $S_{m}^{1}, S_{m}^{2}, \ldots, S_{m}^{k-1}$ состояния $U_{m}=\left\{S_{m}^{0}, S_{m}^{1}, S_{m}^{2}, \ldots, S_{m}^{k-1}\right\}, \quad$ где $m \in\{1,2,3, \ldots, N\}$. Вероятность нахождения СМО в кждом из псевдосостояний зависит в общем случае от времени $t$. Будем обозначать как $p_{m}^{(l)}(t)$ вероятность пребывания системы в состоянии $S_{m}^{l}$, где $l \in\{0,1,2, \ldots, k\}$.

$$
\left\{\begin{array}{l}
\frac{d p_{m}^{(1)}(t)}{d t}=-\lambda p_{m}^{(1)}(t)+\lambda p_{m}^{(0)}(t) \\
\frac{d p_{m}^{(2)}(t)}{d t}=-\lambda p_{m}^{(2)}(t)+\lambda p_{m}^{(1)}(t) \\
\cdots \\
\frac{d p_{m}^{(k-1)}(t)}{d t}=-\lambda p_{m}^{(k-1)}(t)+\lambda p_{m}^{(k-2)}(t)
\end{array}\right.
$$


Обыкновенное дифференциальное уравнение для пребывания СМО в псевдосостоянии $S_{m}^{0}$ при $m \in\{1,2,3, \ldots, N\}$ имеет вид

$$
\frac{d p_{m}^{(0)}(t)}{d t}=-(\lambda+\mu) p_{m}^{(0)}(t)+\lambda p_{m-1}^{(k-1)}(t)+\mu p_{m+1}^{(0)}(t)
$$

Подмножество $U_{N+1}$ состоит из одного псевдосостояния $S_{N+1}^{0}$, поэтому соответствующее дифференциальное уравнение имеет вид

$$
\frac{d p_{N+1}^{(0)}(t)}{d t}=-\mu p_{N+1}^{(0)}(t)+\lambda p_{N}^{(k-1)}(t)
$$

Таким образом, система дифференциальных уравнений Колмогорова для составляемой модели массового обслуживания имеет следующий вид:

$$
\left\{\begin{array}{l}
\frac{d p_{m}^{(1)}(t)}{d t}=-\lambda p_{m}^{(1)}(t)+\lambda p_{m}^{(0)}(t) \\
\frac{d p_{m}^{(2)}(t)}{d t}=-\lambda p_{m}^{(2)}(t)+\lambda p_{m}^{(1)}(t) \\
\cdots \\
\frac{d p_{m}^{(k-1)}(t)}{d t}=-\lambda p_{m}^{(k-1)}(t)+\lambda p_{m}^{(k-2)}(t) \\
\frac{d p_{m}^{(0)}(t)}{d t}=-(\lambda+\mu) p_{m}^{(0)}(t)+\lambda p_{m-1}^{(k-1)}(t)+\mu p_{m+1}^{(0)}(t) \\
\cdots \\
\frac{d p_{N+1}^{(0)}(t)}{d t}=-\lambda p_{N+1}^{(0)}(t)+\lambda p_{N}^{(k-1)}(t)
\end{array}, m \in\{1,2,3, \ldots, N\}\right.
$$

Кроме того справедливо равенство

$$
\sum_{m=0}^{N}\left(\sum_{i=0}^{k-1} p_{m}^{(i)}(t)\right)+p_{N+1}^{(0)}=1
$$

Начальные условия следующие:

$$
p_{0}^{(0)}(0)=1, \quad p_{m}^{(l)}(0)=0 \text { для } m \in\{1,2,3, \ldots, N+1\}, l \in\{0,1,2, \ldots, k\} .
$$

Вероятность того, что в момент времени $t$ на участке улично-дорожной сети $B C$ находится $m$ автомобилей - это вероятность того, что система массового обслуживания пребывает в состоянии $U_{m}$. Эта вероятность $P_{m}$ вычисляется следующим образом:

$$
P_{m}(t)=\sum_{i=0}^{k-1} p_{m}^{(i)}(t), m \in\{1,2,3, \ldots, N\}: P_{N+1}(t)=p_{N+1}^{(0)}(t)
$$

Ситуация, сложившаяся на участке улично-дорожной сети, описывается следующими характеристиками СМO:

1) вероятность того, что в момент времени $t$ данный участок загружен полностью, равна

$$
P_{N+1}(t)=p_{N+1}^{(0)}(t)
$$


2) среднее количество автомобилей на данном участке дороги в момент времени $t$ равно математическому ожиданию числа автомобилей в очереди в данный момент:

$$
M(l(t))=\sum_{i=1}^{N+1} i \cdot P_{i}(t)
$$

здесь $l(t)$ - случайная величина, число автомобилей в очереди в момент времени $t$;

3) среднее время проезда (в секундах) по участку $A C$ улично-дорожной сети равно

$$
M\left(T_{\text {del }}(t)\right)=\frac{\sum_{i=1}^{N+1} i \cdot P_{i}(t)}{\lambda},
$$

здесь $T_{d e l}(t)$ - случайная величина, время проезда по участку $A C$ улично-дорожной сети автомобиля, находящегося в $i$-й позиции в очереди в момент времени $t$.

3. Характеристики движения автотранспортных средств в плотном потоке придорожно-транспортном происшествии

Время функционирования системы массового обслуживания может быть разделено на два временных участка:

$\left(0, \tau_{s t}\right)$ - вероятности пребывания заявки в конкретном состоянии зависят от времени;

$t \in\left(\tau_{s t}, \infty\right)$ - вероятности пребывания заявки в конкретном состоянии не зависят от времени (стационарное состояние).

Если дорожно-транспортное происшествие только что произошло, то следует рассматривать функционирование системы на промежутке $\left(0, \tau_{\mathrm{ss}}\right)$. В данном случае основная трудность состоит в решении системы дифференциальных уравнений (4). Найти точное аналитическое решение возможно лишь при отдельных конкретных значениях параметров $\lambda$ и $\mu$. Поэтому при решении поставленной практической задачи гораздо удобнее найти численное решение системы для заданного момента $t_{1}$. Например, это можно сделать методом Рунге - Кутты. После определения численных значений вероятностей $p_{m}^{(l)}(t)$ в момент $t_{1}$ можно вычислить необходимые показатели функционирования системы по формулам (8)-(10).

Однако, как показывают практические исследования, через достаточно короткое время $\tau_{s t}$ после дорожно-транспортного происшествия, система переходит в стационарное состояние. Поэтому на промежутке $\left(0, \tau_{s}\right)$ можно считать вероятности $p_{m}^{(l)}(t)$ постоянными величинами, не зависящими от времени $t$. Следовательно, их производные по времени равны нулю.
Тогда система (1) принимает вид

$$
\left\{\begin{array}{l}
0=-\lambda p_{m}^{(1)}+\lambda p_{m}^{(0)} \\
0=-\lambda p_{m}^{(2)}+\lambda p_{m}^{(1)} \\
\cdots \\
0=-\lambda p_{m}^{(k-1)}+\lambda p_{m}^{(k-2)}
\end{array}\right.
$$

Отсюда следует, что

$$
\begin{gathered}
p_{m}^{(0)}=p_{m}^{(1)}=\ldots=p_{m}^{(k-1)}, \\
m \in\{1,2,3, \ldots, N\} .
\end{gathered}
$$

Учитывая результат (12) и дифференциальные уравнения (2) и (3), получим систему алгебраических уравнений

$$
\begin{aligned}
& \left\{\begin{array}{l}
0=-\lambda p_{0}^{(0)}+\mu p_{1}^{(0)} \\
0=-(\lambda+\mu) p_{m}^{(0)}+\lambda p_{m-1}^{(k-1)}+\mu p_{m+1}^{(0)} \\
0=-\mu p_{N+1}^{(0)}+\lambda p_{N}^{(k-1)}
\end{array}\right. \\
& \text { для } m \in\{1,2,3, \ldots, N\} \text {. }
\end{aligned}
$$

Обозначим $\alpha=\frac{\lambda}{\mu}$. Решение системы:

$p_{m}^{(0)}=\alpha^{m} p_{o}^{(0)}, m \in\{0,1,2, \ldots, N+1\}$.

Значение $p_{o}^{(0)}$ выразим из условия (5):

$$
\begin{aligned}
& 1=\sum_{m=0}^{N}\left(\sum_{i=0}^{k-1} p_{m}^{(i)}\right)+p_{N+1}^{(0)}= \\
& =\sum_{m=0}^{N}\left(k \alpha^{m} p_{0}^{(0)}\right)+\alpha^{N+1} p_{0}^{(0)} .
\end{aligned}
$$

Таким образом:

$$
p_{0}^{(0)}=\frac{1}{\sum_{m=0}^{N}\left(k \alpha^{m}\right)+\alpha^{N+1}} .
$$

Теперь выразим необходимые характеристики:

1) вероятность того, что данный участок загружен полностью, равна

$$
P_{N+1}=\alpha^{N+1} p_{0}^{(0)}=\frac{\alpha^{N+1}}{\sum_{m=0}^{N}\left(k \alpha^{m}\right)+\alpha^{N+1}} ;
$$


2) среднее количество автомобилей на данном участке дороги $A C$ :

$$
\begin{aligned}
M(l)=\sum_{m=1}^{N}\left(m \cdot\left(\sum_{i=0}^{k-1} p_{m}^{(i)}\right)\right) & +(N+1) \cdot \alpha^{N+1} p_{0}^{(0)}=p_{0}^{(0)} \cdot\left(\sum_{m=1}^{N} m \cdot k \alpha^{m}\right)+(N+1) \alpha^{N+1} p_{0}^{(0)}= \\
& =p_{0}^{(0)} \cdot\left(\left(\sum_{m=0}^{N} m k \alpha^{m}\right)+(N+1) \alpha^{m}\right) .
\end{aligned}
$$

Итак,

$$
M(l)=\left(\left(\sum_{m=0}^{N} m k \alpha^{m}\right)+(N+1) \alpha^{N+1}\right) \frac{1}{\sum_{m=0}^{N}\left(k \alpha^{m}\right)+\alpha^{N+1}}
$$

3) среднее время проезда по участку $A C$ улично-дорожной сети равно

$$
M\left(T_{d e l}\right)=\frac{\left(\left(N \cdot \sum_{i=0}^{k-1} i \alpha^{i}\right)+(N+1)\right)}{\lambda \cdot\left(\sum_{m=0}^{N}\left(k \alpha^{m}\right)+\alpha^{N+1}\right)} .
$$

В модели TIMeR_Mod, разработанной ранее Н.А. Наумовой, информация о распределении потоков по сети содержится в матрицах $A_{\text {STREETS }}$ и $B_{\text {INTERSECTION }}[6]$. Характеристики (16) $-(18)$ позвоЛяют разработать алгоритм корректировки данных о транспортных потоках при ДТП:

a) если $P_{N}+P_{N+1} \geq 0,9$ или $M(l) \approx N$, то «исключаем» движение по рассматриваемому участку улично-дорожной сети в матрицах $A_{\text {STREETS }}$ и $B_{\text {INTERSECTION }}$ (вводИм запрет на движение соответствующим образом, изложенным в работе [6]);

б) если $P_{N}+P_{N+1}<0,9$ и $M(l)<N-1$ , то уменьшаем количество полос движения в месте инцидента в матрицах $A_{\text {STREETS }}$ $B_{\text {INTERSECTION }}$ и корректируем параметры Эрланга на полосах движения;

в) обновляем OD-матрицу согласно изменениям, внесенным $A_{\text {STREETS }}$ и $B_{\text {INTERSECTION }}$ [7].

Разработанная модель движения автомобилей в случае сужения проезжей части по причине ДТП или дорожно-ремонтных работ позволяет оперативно корректировать организацию движения.

\section{Заключение}

Моделирование движения транспортных потоков вблизи мест дорожно-транспортных происшествий или ремонтных работ позволяет прогнозировать вызванные этим задержки транспортных средств и корректировать оптимальные схемы организации движения в этих ситуациях. В данной работе применялись методы мезоскопического моделирования, отличительной осо- бенностью которых является оперативность выполнения расчетов при соблюдении необходимой точности. Результаты данной работы дополняют модель движения транспортных потоков TIMeR_Mod, разработанную ранее автором настоящей статьи.

\section{Список литературы}

1. Moutari S., Herty M., Klein A., Oeser M., Steinauer B., Schleper V. Modelling road trafficaccidents using macroscopic second-order models of traffic flow. IMA J. Appl. Math. 2013. Vol. 78. P. 1087-1108.

2. Göttlich S., Knapp S. Modeling random traffic accidents by conservation laws. Mathematical Biosciences and Engineering. 2020. Vol. 17 (2). P. 1677-1701. DOI: 10.3934/ mbe. 2020088 .

3. Jin L., Amin S. Analysis of a stochastic switching model of freeway traffic incidents, IEEE Trans. Automat. Control. 2019. Vol. 64. P. 1093-1108.

4. Ockendon H., Mason D.P., Fanucchi D., la Foy T., Oliphant T., Khalique M. A mathematical model of a road block, Mathematics-in-Industry Case Studies Journal. 2010. Vol. 2. P. 134-154.

5. Dymski N., Goatin P., Rosini M. Modeling moving bottlenecks on road networks. Hyperbolic Problems: Theory, Numerics, Applications. Proceedings of the XVII international conference in Penn State., PennState, United States. 2018. P. 419-426.

6. Naumova N.A., Danovich L.M. Modelling And Optimisation Of Flows Distribution In The Network, Applied Mathematics. 2012. Vol. 2(5). P. 171-175. DOI: 10.5923/j. am.20120205.04.

7. Naumova N.A. Method For Estimating An Origin-Destination Matrix For Dynamic Assignment, International Journal of Control Theory and Applications. 2016. Vol. 9 (30). P. 129-138.

8. Вентцель Е.С., Овчаров Л.А. Теория случайных процессов и ее инженерные приложения: учебное пособие, 5-е изд., стер. М.: КНОРУС, 2016. 448 с.

9. Cox D.R., Smith W.L. Queues. London: Methuen, P. $150,1961$.

10. Наумова Н.А., Данович Л.М., Данович Ю.И. Определение параметров распределения обобщенного закона Эрланга по экспериментальным данным при изучении транспортных потоков// Современные проблемы науки и образования. 2013. № 5. [Электронный ресурс]. URL: https://science-education.ru/ru/article/view?id=10045 (дата обращения: 01.09.2021). 